\title{
Interfacial chiral selection by bulk species
}

\author{
Hua Dong ${ }^{1}$, Jordi Ignés-Mullol ${ }^{1}$, Josep Claret ${ }^{1}$, Lourdes Pérez ${ }^{2}$, Aurora Pinazo ${ }^{2}$, \\ Francesc Sagués ${ }^{1}$ \\ 1. Departament de Química Física, Universitat de Barcelona, Martí i Franquès 1 \\ E-08028 Barcelona, Catalunya, Spain \\ 2. Institut de Química Avançada de Catalunya, IQAC-CSIC, Jordi Girona 18-26 \\ E-08034 Barcelona, Catalunya, Spain
}

January 20, 2014

\begin{abstract}
In this paper we report on a chiral selection process in a self-assembled soft monolayer of an achiral amphiphile as a consequence of its interaction with chiral species dissolved in the aqueous subphase. The extent of the chiral selection is statistically measured in terms of the enantiomorphic excess of self assembled submillimeter domains endowed with well defined orientational chirality that is unambiguously resolved using optical microscopy. Our results show that the emergence of chirality is mediated by electrostatic interactions and significantly enhanced by hydrophobic effects. This chiral chemical effect can be suppressed and even reversed by opposing a macroscopic physical influence such as vortical stirring. This result evidences the crucial role of hydrodynamic effects in supramolecular aggregation.
\end{abstract}

\section{Introduction}

The emergence of supramolecular chirality is a fundamental and intriguing process related to the origin of the biological homochirality $[1,2]$. In particular, the formation of chiral motifs on a surface could result in a pattern that might be further used to imprint chirality to other systems [3] or as a chiral heterogeneous catalyst [4]. It is thus relevant to analyze the role of different chiral influences on the assembly of chiral structures at well defined interfaces. For this purpose, it is convenient to use a model system, simple to characterize, unambiguously resolved and easy to control, on which to test the effects of chiral forces of different nature.

Langmuir monolayers of some achiral azobenzene derivatives match the requirements for such a quasi two-dimensional system [5]. The key feature of the azobenzene group is its cis-trans photoisomerization. The cis-isomer is stable under UV irradiation and slowly relaxes to the trans conformation under room light. The different polarity of both isomers plays a crucial role in their interfacial activities [6]. The bent cis isomer is polar and forms isotropic monolayers, whereas stretched and apolar trans molecules form ordered domains favoured by H-aggregation $[7,8]$. Under well defined experimental conditions, mixed cis-trans monolayers of 4-[4-[(4-octylphenyl)azo]phenoxy]butanoic acid, $8 \mathrm{Az} 3 \mathrm{COOH}$ in our shorthand notation (Fig. 1), display birefringent trans circular domains embedded in an isotropic pool of cis isomer $[8,9]$. The important point is that after spreading an initially isotropic and featureless cis monolayer, it slowly evolves through the cis $\rightarrow$ trans conversion, leading to the formation of trans elliptical domains by means of a nucleation and growth mechanism limited by the isomerization reaction. Further evolution of the monolayer through coalescence of elliptical domains results in the formation of circular domains. Brewster Angle Microscopy (BAM) [10,11] images unambiguously indicate a tangential orientation of the molecular azimuth inside the circular domains with a central organizing defect, i. e., a bend-like configuration [8]. In absence of any chiral influence, domains display either enantiomorphic orientations 


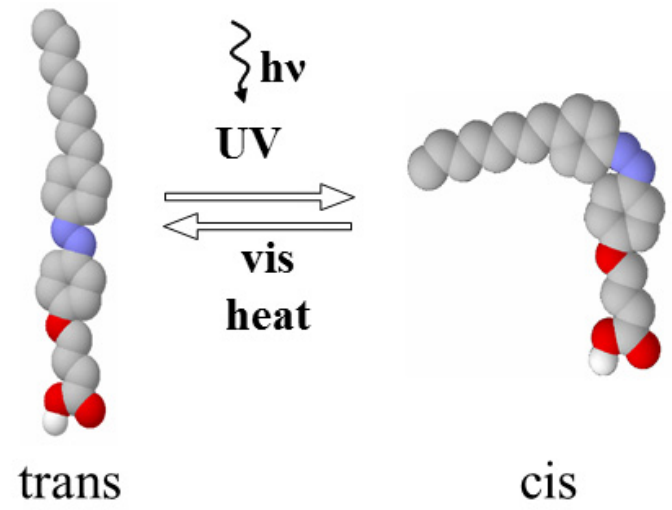

Figure 1: Chemical structure of the isomers of 4-[4-[(4-octylphenyl)azo]phenoxy]butanoic acid $(8 \mathrm{Az} 3 \mathrm{COOH})$. UV irradiation stabilizes the cis isomer that slowly changes into the trans one under room light.

of the hydrophobic tails, clockwise $(\mathrm{CW})$ or counterclockwise $(\mathrm{CCW})$, structuring the monolayer as a two-dimensional racemic mixture [12].

The presence of chiral influences during the evolution of a cis monolayer breaks the balance of CW and CCW domains. Actually, it has been demonstrated by our group [13] that the application of a physical chiral influence as represented by a subphase vortical stirring [1] permits to select the statistical chirality of the monolayer. Clockwise (counterclockwise) vortical stirring of the aqueous subphase leads to an excess of $\mathrm{CW}(\mathrm{CCW})$ bend-like circular domains. This vortical chiral force acts during the merging process of primordial elliptic domains through a complex process of generation and annihilation of topological defects, as discussed elsewhere [13].

A chiral influence of a very different nature arises from the presence of amphiphilic chiral dopants in the monolayer [14]. We have observed that, after spreading a cis-8Az3COOH solution with small amounts $(\approx 6 \%)$ of an enantiomer of a chemically related chiral azobenzene derivative, an excess of one of the two enantiomorphic bend domains is found. Moreover, in this case, the chiral dopant modifies the shape of primordial domains since asymmetric bean-like domains are found instead of elliptical ones. Coalescence of these domains also results in CW or CCW bend structures [14].

Vortical stirring is a bulk chiral force of physical nature acting at the centimeter scale, whereas the presence of the chiral dopant, a chemical influence, affects directly the spread monolayer at the nanoscale. In spite of these different origins and operating length scales, both chiral forces lead strikingly to qualitatively similar results, and can indeed compete to select an enantiomeric excess in a $8 \mathrm{Az} 3 \mathrm{COOH}$ Langmuir monolayer. The mechanism of this chiral competition can be explained on the basis of a kinetic mechanism that combines the concurrent effects of the two chiral forces as if they were acting separately [14].

Another chiral influence we can envisage, different from those just mentioned, is the presence of chiral compounds in the subphase such as amino acids. Chiral influences of different amino acids on supramolecular structures in various experimental scenarios have been reported in the literature. For instance, amino acid enantiomers prompt the formation of chiral supramolecular aggregates from achiral building blocks in bulk solution $[15,16,17]$. On the other hand, different effects of amino acids on adsorbed species at interfaces of different nature have also been reported [18, 19, 20]. In particular, chiral interactions of water soluble amino acids and different Langmuir monolayers have also been found $[21,22,23]$.

In this paper, we report on the effect of water soluble chiral amino acid on 8Az3COOH Langmuir monolayers. The chiral influence of amino acids will be analyzed in terms of their structural and hy- 
drophobic characteristics. Finally, the effect of ionic strength on this chiral selection and its competition with vortical stirring of the subphase will be addressed.

\section{Experimental}

\subsection{Materials}

The synthesis of $8 \mathrm{Az} 3 \mathrm{COOH}$ was reported previously [9]. The aqueous subphase is an amino acid solution in ultrapure water (resistivity $\approx 18.2 \mathrm{M} \Omega \mathrm{cm}$ ) supplied by a Millipore MilliQ system unless otherwise stated. Spreading solutions $(\approx 1 \mathrm{mM}$ of the cis isomer) were prepared using chloroform (Baker p.a.) as solvent. The amino acids were supplied by Sigma and used as received. In order to check the effect of ionic strength $(I)$ on the chiral selection process, appropriate amounts of $\mathrm{NaCl}$ (Merck p.a.) were added to the amino acid solution. Amphiphilic amino acids, N-Acyl-Arginine methyl ester hydrochlorides, were synthesized as described in reference [24].

\subsection{Preparation and observation of the monolayers}

In a typical experiment, monolayers are prepared by depositing the cis-based spreading solution on an aqueous solution of the selected amino acid contained in a Langmuir cuvette under room light up to a surface pressure in the range $0-1 \mathrm{mN} \mathrm{m}^{-1}$. Temperature of the subphase is kept constant at $20 \pm 0.5^{\circ} \mathrm{C}$ by means of a re-circulating bath. Thirty minutes after spreading, when the trans elliptical domains have evolved, the pressure is increased up to $2 \mathrm{mN} \mathrm{m}^{-1}$ to favor their fusion into the chiral bend domains. Representative BAM images of the monolayer are recorded during $30 \mathrm{~min}$ afterwards. The extension of the handedness selection is quantified as the enantiomeric excess of $\mathrm{CW}$ domains, $e e_{C W}$, defined as

$$
e e_{C W}=\frac{n_{C W}-n_{C C W}}{n_{T}} 100
$$

where $n_{T}$ is the number of analyzed bend domains, and $n_{C W}$ and $n_{C C W}$ represent the number of CW and CCW bend domains, respectively. Values of $e e_{C W}$ have been calculated for $n_{T}$ within the range of 700 - 800 domains in each measurement from observations corresponding to different locations in the monolayer at different times. Therefore, the resulting $e_{C W}$ value can be considered as an average over the monolayer during the recording time.

In order to study the effect of vortical stirring, a black Delrin circular cell, $13 \mathrm{~mm}$ of depth and $60 \mathrm{~mm}$ of diameter, is placed in the Langmuir trough. The subphase contained in the cell is stirred at a constant rate $\Omega$ by means of a reversible magnetic stirrer (J. P. Selecta, Spain) with a submerged cylindrical Teflon coated magnetic bar ( $8 \mathrm{~mm}$ long, $3 \mathrm{~mm}$ diameter). Monolayers are prepared by depositing at the outer part of the vortical funnel one drop $(2 \mu \mathrm{l})$ of the spreading solution.

Imaging of the quiescent monolayer is performed by means of a custom-built Brewster angle microscope (BAM). The relationship between the azimuth of the azobenzene molecules in the domain and the reflectivity of BAM images has been reported previously [9]. BAM images are analyzed by means of the public software ImageJ [26].

\section{Experimental results and discussion}

The investigated amino acids are shown in Table 1. As a preliminary test, we have checked the interfacial activity of a particular representative amino acid at the pure water surface under our particular experimental conditions. Phenylalanine (hydropathy index of 2.8), an essential amino acid bearing an hydrophobic side chain due to the presence of a benzene ring, has been chosen for this purpose. A sig-

nificant decrease of the surface tension of the air-water interface is not observed after injection of L-Phe to the subphase up to the concentration range studied in this work. 
BAM images show that, at low amino acid concentrations, the general texture of the monolayer and its initial evolution after spreading the $\mathrm{cis}-8 \mathrm{Az} 3 \mathrm{COOH}$ chloroform solution is similar to that observed on a pure water subphase (Fig. 2) [8,9]. The most significant difference is an increase in the lability of the trans domains with the concentration of the amino acid that results in the complete loss of birefringent structures at high enough concentrations. This suggests that a possible $\pi-\pi$ interaction between the aromatic groups of 8-Az3COOH and L-Phe may occur giving rise to non-birefringent aggregates. Similar results were obtained for hexadecanoil phenylalanine [27]. However, a neat chiral symmetry breaking is unambiguously detected at concentration values up to $10-15 \mathrm{mg} \mathrm{l}^{-1}$.
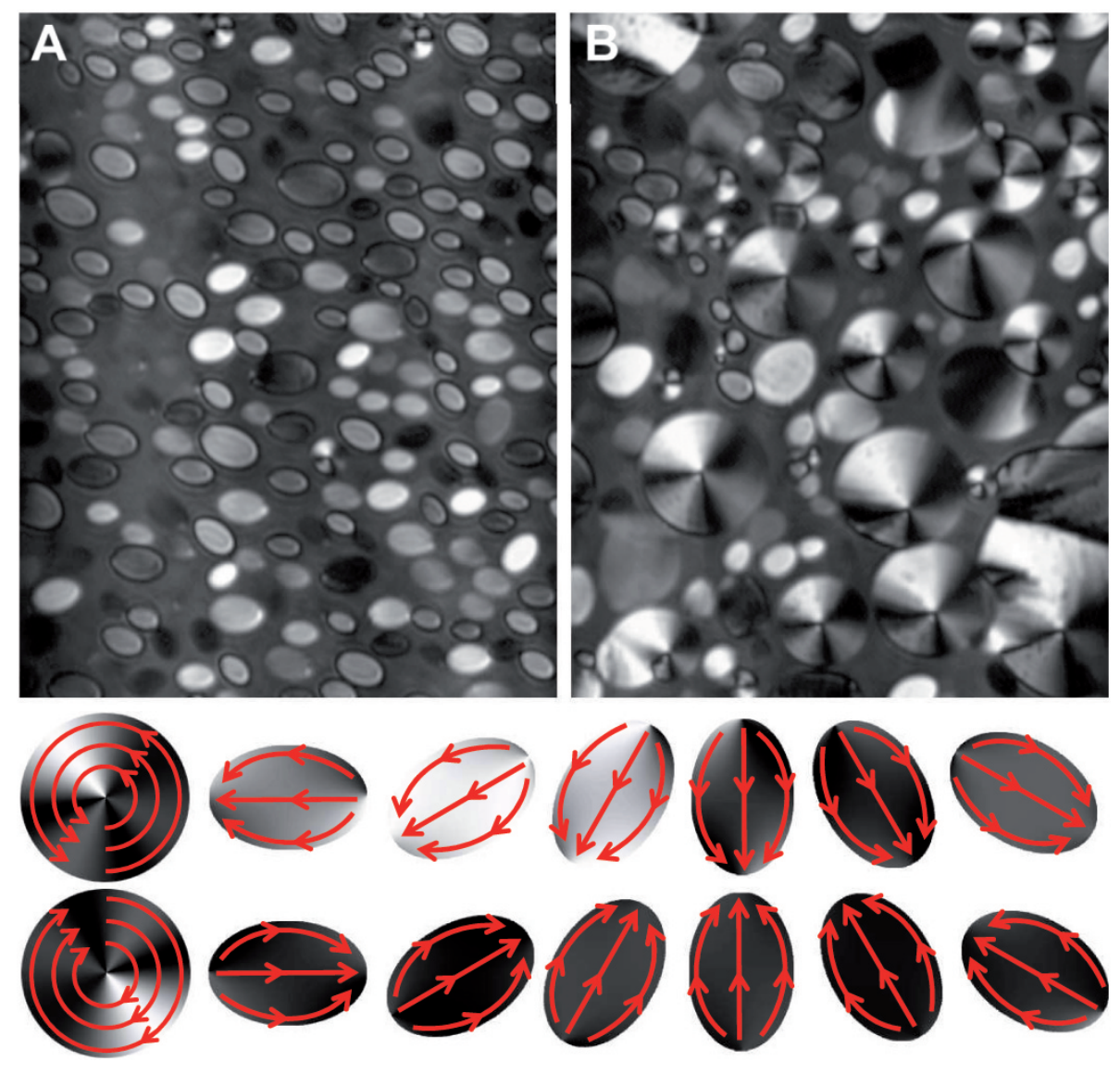

Figure 2: Typical BAM images of an initially cis-8Az3COOH monolayer on a L-Phe solution of $\approx 1 \mathrm{mg}$ $\mathrm{l}^{-1}$ at $1 \mathrm{mN} \mathrm{m}^{-1}, 40 \mathrm{~min}$ after spreading (A), and at $2 \mathrm{mN} \mathrm{m}^{-1}, 35 \mathrm{~min}$ after spreading (B). Notice the prevalence of $\mathrm{CCW}$ bend domains in front of $\mathrm{CW}$ ones in panel $\mathrm{B}$. The analyzer is set at -74 with respect to the plane of incidence, which contains the $y$-axis of the images. $T=19.9^{\circ} \mathrm{C}$. The width of the image is $550 \mu \mathrm{m}$. Below: correlation between the gray scale BAM image and the azimuth of the local molecular orientation for elliptic and circular bend-like domains [9].

Figure 3 shows the effect of the concentration of L-Phe on the balance of CW and CCW bend domains. Even at a concentration as low as $10 \mu \mathrm{g} \mathrm{l^{-1 }}$, an excess of CCW bend domains, $e e_{C W} \approx-16$, is clearly observed. Increasing concentration, the enantiomeric excess of CCW domains attains a maximum value, $e e_{C W} \approx-37$, at c.a. $1 \mathrm{mg} \mathrm{l}^{-1}$. Notice that a complete symmetry breaking is never achieved consistently with what was observed for chiral influences studied earlier for 8Az3COOH Langmuir monolayers $[12,14]$. This is a consequence of the kinetic control of the chiral selection mechanism [13], a general feature of the chiral selection process of azobenzene interfacial assemblies, that will be discussed in the last section for this particular situation. In view of this result, $1 \mathrm{mg} \mathrm{l}^{-1}$ has been chosen as the working concentration of the amino acids in the aqueous subphase unless otherwise stated. The expected reversal in the chiral 
selection when changing the chirality of the dopant is evidenced since $e_{C W} \approx 32$ is obtained when cis-8Az3COOH is spread on a solution of D-Phe at $1 \mathrm{mg} \mathrm{l}^{-1}$.

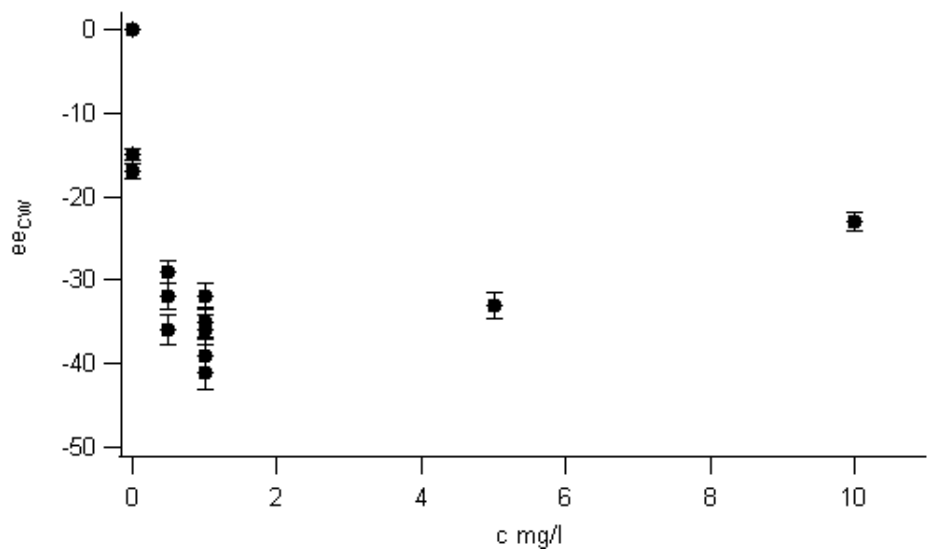

Figure 3: Dependence of the enantiomeric excess of CW domains on L-Phe concentration.

When the aqueous subphase contains L-Ala (hydropathy index 1.8) or L-Ile (hydropathy index 4.5), azobenzene monolayers follow a similar behavior as reported above but the enantiomeric excess is significantly lower than for L-Phe, $e e_{C W} \approx-30$ for L-Ala and $e e_{C W} \approx-32$ for L-Ile. This result points out a lack of correlation between the hydrophobicity of the amino acid and its interaction with the azobenzene monolayer, leading to the chiral symmetry breaking, . This suggests that, in spite that the hydrophobic character of the amino acid should play a relevant role in the chiral selection of the soft monolayer, other effects due to the specific structure of the amino acid should be taken into account. This will be confirmed and discussed after studying hydrophilic amino acids.

\subsection{Hydrophilic amino acids: arginine, asparagine, serine, tyrosine}

Hydrophilic amino acids also modify the balance of CW and CCW domains. L-Arg, L-Asn, L-Tyr and L-Ser with hydropathy indexes - 4.5, - 3.5, -1.3 and - 0.8, respectively, have been tested. The general trends of the $8 \mathrm{Az} 3 \mathrm{COOH}$ monolayer are the same as for hydrophobic amino acids, i.e., L-amino acids promote the formation of $\mathrm{CCW}$ bend domains, but the enantiomeric excesses are slightly lower, $e e_{C W} \approx$ $-26, e e_{C W} \approx-28$ and $e e_{C W} \approx-27$ for L-Asn, L-Arg and L-Ser, respectively. The enantiomeric excess due to tyrosine, which also bears a benzene ring, $e e_{C W} \approx-32$, is larger than those of the other hydrophilic amino acids tested. This is similar to the behavior of L-Phe within the series of hydrophobic amino acids. As expected, D-Arg leads to a reverse excess of $e e_{C W} \approx 26$.

Figure 4 summarizes the dependence of enantiomeric excess on the hydropathy index for the studied amino acids. This plot shows a neat chiral symmetry breaking due to the amino acid moiety that increases with the hydrophobicity of the side chain. Actually, besides this hydrophobic effect, $e e_{C W}$ values are also influenced by the chemical structure of the side chain. The presence of a benzene ring significantly enhances the chiral influence suggesting a more specific and closer (stronger) interaction of an aromatic chain with the $8 \mathrm{Az} 3 \mathrm{COOH}$ Langmuir monolayer.

\subsection{Amphiphilic amino acids}

We have also studied the effect of amphiphilic compounds bearing an asymmetric carbon similar to that of amino acids. The L-enantiomers of two N-acyl-Arginine methyl ester derivatives have been selected (Fig. 5). For this purpose, a chloroform solution of cis-8Az3COOH $(\approx 1 \mathrm{mM})$ containing $3 \%$ of the amphiphile is spread on a water subphase, and the evolution of the monolayer is analyzed as described 


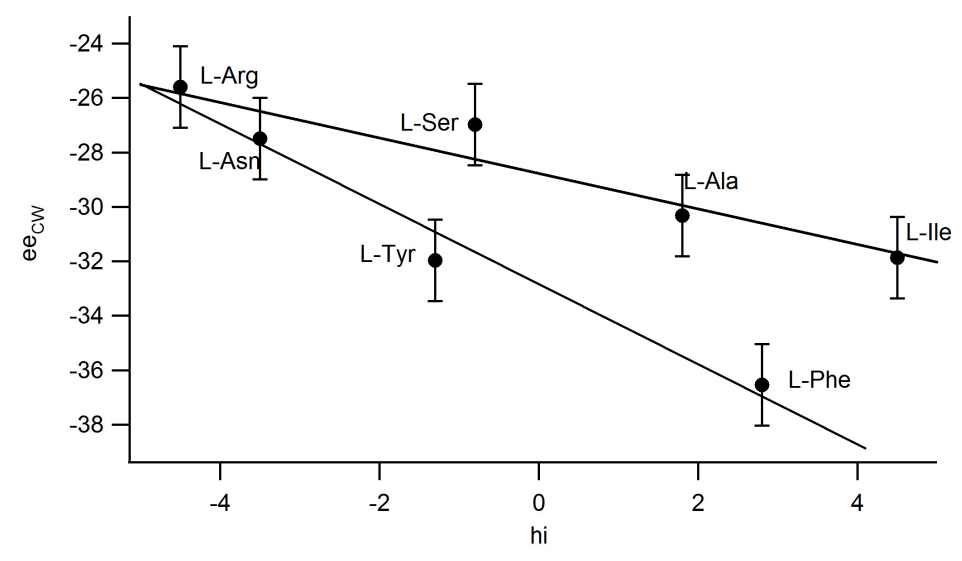

Figure 4: Dependence of the enantiomeric excess of CW domains on the hydropathic index, hi, of the amino acid side chain.

in section 2. Experimental results are qualitatively the same as those found for the soluble amino acids, the formation of CCW bend domains being also favoured in front of $\mathrm{CW}$ ones. The extent of the chiral selection is smaller in this case, $e e_{C W} \approx-16$ and $e e_{C W} \approx-25$ for MAM and PAM (see caption of Fig. $5)$, respectively. We can observe that $e e_{C W}$ increases with the length of the hydrophobic tail, i.e., the hydrophobicity of the amphiphile, a similar trend to that shown by the soluble amino acids.

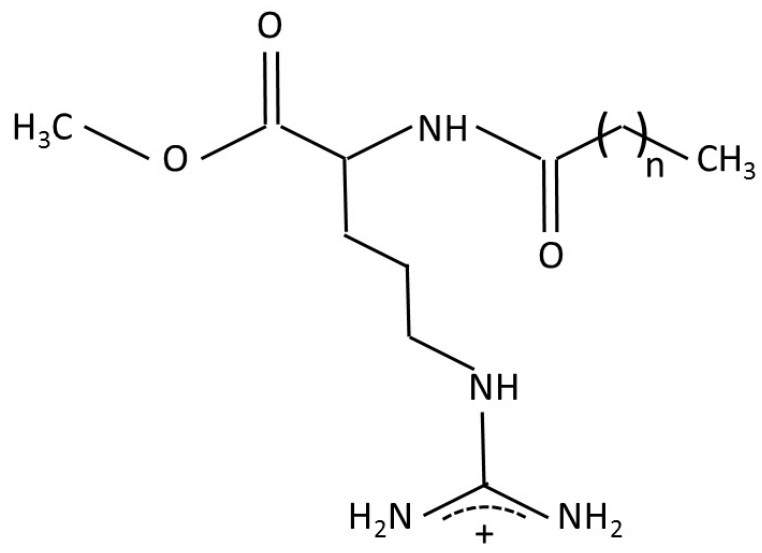

$\mathrm{Cl}^{-}$

Figure 5: Amphiphilic arginine derivatives: N-myristoyl-Arginine methyl ester $(n=12$, MAM) and N-palmitoyl-Arginine methyl ester $(n=14$, PAM) hydrochlorides.

Two more results are worth to mention in this latter situation. The chiral amphiphile does not modify the shape of the elliptical primordial domains formed during the evolution of the initial cis- $8 \mathrm{Az} 3 \mathrm{COOH}$ monolayer. This contrasts with the effect of chiral amphiphilic azobenzene derivatives reported previously where chiral bean-like domains are mostly formed instead of achiral elliptical ones [14]. Another remark refers to the effect of increasing the amphiphile concentration in the monolayer. When the monolayer contains $6 \%$ of MAM, circular bend domains are no longer formed and the monolayer only displays elliptical domains.

Since MAM and PAM self assemble in aqueous solution forming elongated aggregates like cylindrical or ellipsoidal micelles [28], the coexistence of these compounds with $8 \mathrm{Az} 3 \mathrm{COOH}$ at the air-water interface 
would prompt synergistic effects inducing the formation of more stable elongated domains than those observed in a pure $8 \mathrm{Az} 3 \mathrm{COOH}$ monolayer. Moreover, the absence of coalescence of these elliptical domains at large concentrations of the amphiphilic amino acids suggests that they play an interfacial role, preferably adsorbing at the boundary line of these domains, lowering their line tension, i. e., acting as a linactant [29], and preventing domain coalescence that would lead to the formation of circular domains.

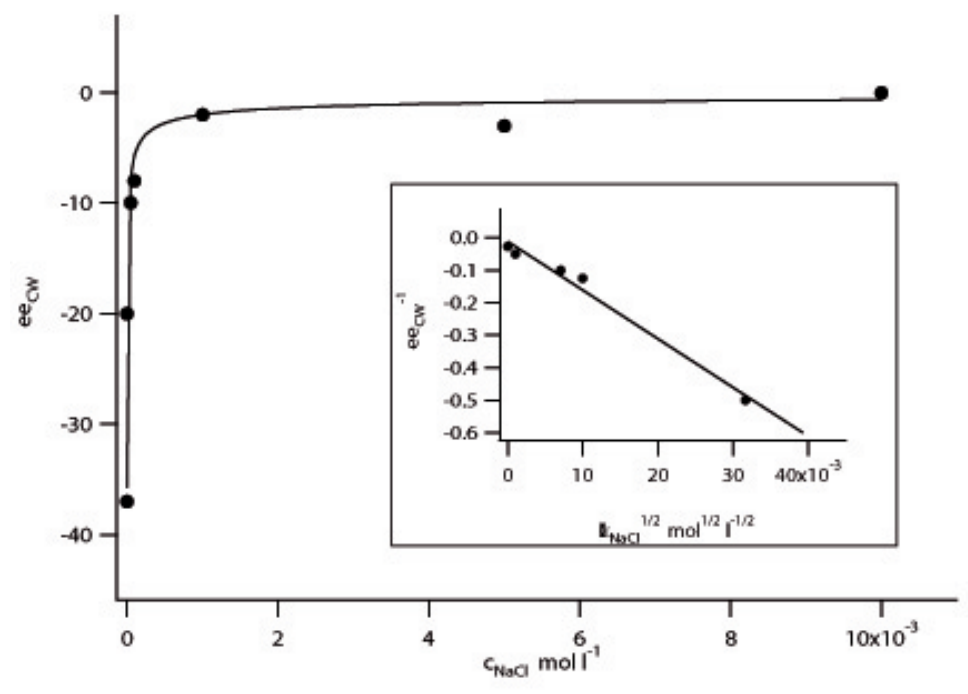

Figure 6: Dependence of the enantiomeric excess of $\mathrm{CW}$ domains on $\mathrm{NaCl}$ concentration. Inset shows a linear dependence of $e e_{C W}^{-1}$ on the square root of $N a C l$ concentration for the initial range up to $10^{-3} \mathrm{M}$. $c_{L-P h e}=1 \mathrm{mg} \mathrm{l}^{-1}$.

\subsection{Effect of ionic strength of the subphase}

In order to check the relevance of electrostatic interactions on the chiral selection due to soluble amino acids, we have performed a series of experiments changing the ionic strength of the aqueous subphase. We have found that 1:1 electrolytes, $\mathrm{NaCl}$ and $\mathrm{CsCl}$, in the subphase up to concentrations of $0.1 \mathrm{M}$ increase the lability of the trans-domains but the monolayer retains the same general trends as on pure water. On the other had, even dilute solutions, $\simeq 10^{-5}$, of $2: 1$ electrolytes, $Z n C l_{2}$ and $C d C l_{2}$, strongly modify the morphology of the $8 \mathrm{Az} 3 \mathrm{COOH}$ Langmuir monolayer and well defined bend domains are scarcely observed.

In a series of experiments, $e e_{C W}$ has been measured for monolayers spread on a $1 \mathrm{mg} \mathrm{l^{-1 }}$ solution of L-Phe with different $\mathrm{NaCl}$ concentrations. The results are summarised in Fig. 6. We observe that $e e_{C W}$ decreases by fifty percent for $C_{\mathrm{NaCl}} \simeq 10^{-6} \mathrm{M}$ and vanishes for $c_{\mathrm{NaCl}}>10^{-3} \mathrm{M}$.

According to these results, we conclude that electrostatic interactions between dissolved amino acids and the azobenzene surfactant in the monolayer play an important role in the chiral selection process.

\subsection{Effect of vortical stirring}

The effect of vortical stirring on chiral selection in soft matter systems has been evidenced in bulk systems [30] and in Langmuir monolayers $[12,13]$. As mentioned in the Introduction, we have recently reported that chiral selection due to an amphiphile dopant in the monolayer can be counterbalanced by vortical stirring [14]. A similar scenario has been investigated here by applying a CW stirring to the subphase containing L-Phe under the same experimental conditions as reported above for two values of the stirring 
rate, $\Omega=100$ and $1000 \mathrm{rpm}$. This arrangement opposes chemical and physical influences since CW stirring selects $\mathrm{CW}$ bend domains whereas L-amino acids promote CCW domains.

The enantiomeric excess of $\mathrm{CW}$ domains in absence of stirring, $e e_{C W} \approx-36$, slightly diminishes under stirring at $100 \mathrm{rpm}\left(e e_{C W} \approx-32\right)$ and is completely reversed at rotating rates above $\Omega=1000 \mathrm{rpm}$. This result is consistent with the one obtained in our group with a chiral dopant in the monolayer [14].

\section{Mechanism of the chiral selection process}

From our previous studies we know that the chiral interaction leading to unbalanced amounts of CW and $\mathrm{CCW}$ bend domains acts during their formation process from an isotropic cis-8Az3COOH monolayer $[12,13,14]$.

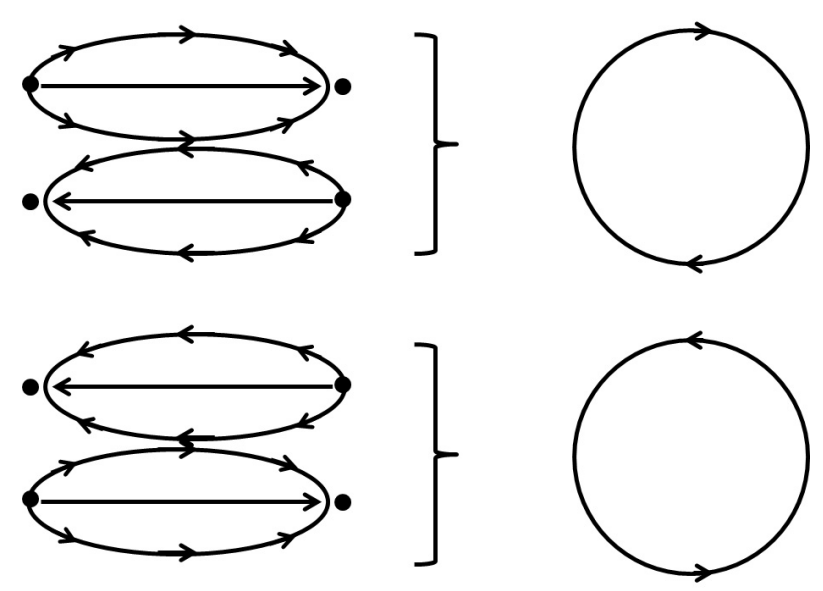

Figure 7: Schematic representation of the molecular azimuth of antiparallel elliptical domains and their fusion leading to $\mathrm{CW}$ and $\mathrm{CCW}$ bend-like circular domains.

As mentioned in the Introduction, when a cis monolayer is left under room light, the cis $\rightarrow$ trans isomerization takes place leading to the formation of axisymmetric elliptical domains. Prompted by face to face aggregation ( $\mathrm{H}$ aggregation) of newly formed trans-8Az3COOH molecules, elliptical domains nucleate, embedded in an isotropic cis pool, with an orientation of the hydrophobic chains parallel, on average, to the long axis of the ellipse (Fig. 2) .

The second step is the coalescence of two elliptical domains. If the fusion takes place between parallel ellipses, the resulting domain is larger with the same characteristics of the parent ones. However, the coalescence of two antiparallel ellipses leads to the formation of a circular bend-like domain (Fig. 7). The combination of orientational order of the surfactant molecules inside the elliptical domain and the curved boundary leads to two topological singularities, i.e. highly distorted structures, at the poles in the form of point defects. Moreover, two additional defects appear at the contact points when two antiparallel domains merge. It is the dynamics of annihilation of these defects which sets the kinetics of chiral domain formation. In particular, defects formed upon ellipse coalescence move to combine with the highest distortion point on the original ellipse, found at the pole from which hydrophobic tails tilt. Defect motion is along the boundary in clockwise (counterclockwise) direction when the fusion leads to a CW (CCW) bend domain (see figure 8). Afterwards, the two remaining initial defects enter into the fused domain and merge leading to the final bend domain, CW or CCW [13].

As explained above, the presence of amino acids in the subphase does not affect the shape of the domains formed after the cis $\rightarrow$ trans isomerization. Therefore, their chiral influence must affect the fusion of two antiparallel domains. Notice that the topological defects formed after two antiparallel elliptical domains contact are chiral, and they move following chiral trajectories, CW or CCW, to form 


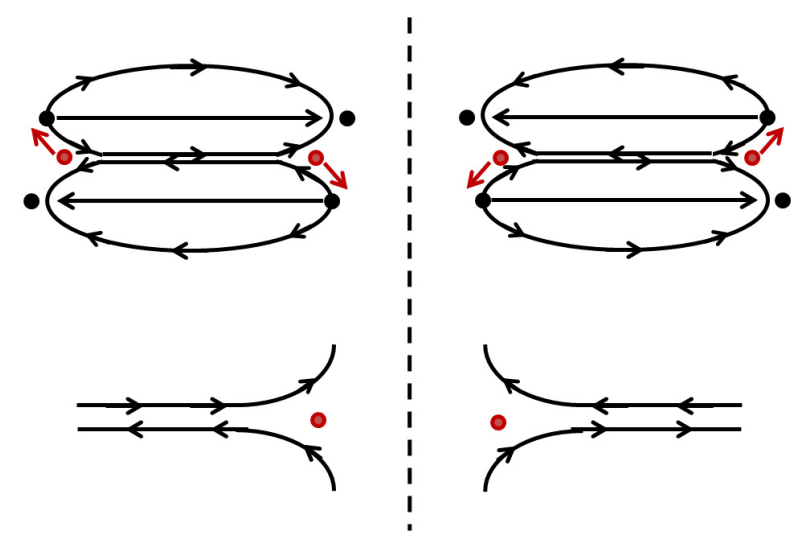

Figure 8: Schematic representation of the molecular azimuth after contact of antiparallel elliptical domains and the chirality of the two newly formed defects.

the final bend domain (Fig. 8). Then, it is reasonable to expect that amino acid enantiomers in the subphase could modify the dynamics of this process. Our results strongly suggest that L-amino acid enantiomers increase the velocity of the defect motion along the boundary of the merging domains in the counterclockwise direction. Unfortunately, our attempts to measure this velocity asymmetry have been inconclusive to quantitatively prove this rationale.

In any case, the interaction responsible for this behavior involves some different contributions. The strong dependence of $e e_{C W}$ on the ionic strength of the subphase (Sec. 3.3) points out a relevant influence of ionic interactions. In our subphase conditions, $p H \approx 6-6.5$, zwitterion is the predominant form of the amino acid molecule. We can thus conclude that the electrostatic interaction between the protonated amino groups of the amino acid molecules with the carboxylate heads of trans-8Az3COOH at the water interface is primarily responsible for the chiral influence. This is further enhanced by hydrophobic interactions, presumably between the amino acid side chain and the hydrophobic tail of the amphiphilic azobenzene derivative. This is supported by the larger effect of amino acids with a benzene ring in its side chain (Tyr and Phe). We can hypotesize on an eventual interaction between the aromatic moieties of these amino acids and the azobenzene derivative at the air-water interface.

Finally, it is worth recalling that these chiral chemical effects acting at the nanoscale are overridden and reversed by a macroscopic physical force acting on a centimeter scale like vortical stirring of the subphase. This supports the crucial role played by hydrodynamics in supramolecular organization [31] as pointed in the pioneering work by Ribó and coworkers on supramolecular aggregation of some porphyrin derivatives in aqueous solutions [30].

\section{Acknowlegment}

We acknowledge funding from MICINN (Project No. FIS2010-21924-C02-01), MINECO (Projects No. CTQ2010-14897 and MAT2012-38047-CO-02) and DURSI, Generalitat de Catalunya (Projects No. 2009SGR-1055 and 2009-SGR-1331).

\section{References}

[1] A. Guijarro and M. Yus. The origin of Chirality in the Molecules of Life. A Revision from Awareness to the Current Theories and Perspectives of this Unsolved Problem. RSC Publishing, Cambridge, UK, 2009. 
[2] I. Weissbuch and M. Lahav. Crystalline architectures as templates of relevance to the origins of homochirality. Chemical Reviews, 111(5):3236-3267, 2011.

[3] I. Weissbuch, L. Leiserowitz, and M. Lahav. Molecular recognition in chiral and non-chiral amphiphilic self-assemblies at interfaces. Current Opinion in Colloid $\&$ Interface Science, 13(1-2):12-22, 2008 .

[4] R. Raval. Nanoscale insights into the creation of chiral surfaces. Journal of Molecular Catalysis a-Chemical, 305(1-2):112-116, 2009.

[5] G. L. Gaines. Insoluble Monolayers at Liquid-Gas Interfaces. Intersciences Publishers, New York, 1966.

[6] D. J. W. Bullock, C. W. N. Cumper, and A. I. Vogel. Physical properties and chemical constitution. part xliii. the electric dipole moments of azobenzene, azipyridines, and azoquinolines. Journal of The Chemical Society, pages 5316-5323, 1965.

[7] J. M. Pedrosa, M. T. Martín-Romero, L. Camacho, and D. Möbius. Organization of an amphiphilic azobenzene derivative in monolayers at the air-water interface. Journal of Physical Chemistry B, 106(10):2583-2591, 2002.

[8] J. Ignés-Mullol, J. Claret, R. Albalat, J. Crusats, R. Reigada, M. T. Martín-Romero, and F. Sagués. Texture changes inside smectic-c droplets in azobenzene langmuir monolayers. Langmuir, 21(7):29482955, 2005.

[9] J. Crusats, R. Albalat, J. Claret, J. Ignés-Mullol, and F. Sagués. Influence of temperature and composition on the mesoscopic textures of azobenzene langmuir monolayers. Langmuir, 20(20):86688674, 2004.

[10] S. Hénon and J. Meunier. Microscope at the brewster-angle: Direct observation of 1st-order phasetransitions in monolayers. Review of Scientific Instruments, 62(4):936-939, 1991.

[11] D. Hönig and D. Möbius. Direct visualization of monolayers at the air-water-interface by brewsterangle microscopy. Journal of Physical Chemistry, 95(12):4590-4592, 1991.

[12] N. Petit-Garrido, J. Claret, J. Ignés-Mullol, J. A. Farrera, and F. Sagués. Chiral-symmetry selection in soft monolayers under vortical flow. Chemistry-a European Journal, 18(13):3975-3980, 2012.

[13] N. Petit-Garrido, J. Claret, Ignés-Mullol J., and F. Sagués. Chiral selection by interfacial shearing of self-assembled achiral molecules. Physical Review Letters, 103:237802, 2009.

[14] N. Petit-Garrido, J. Claret, Ignés-Mullol J., and F. Sagués. Stirring competes with chemical induction in chiral selection of soft matter aggregates. Nature Communications, 3:1001, 2012.

[15] R. Lauceri, A. D'Urso, A. Mammana, and R. Purrello. Chiral memory: Induction, amplification, and switching in porphyrin assemblies. Chirality, 20(3-4):411-419, 2008.

[16] L. X. Zeng, Y. J. He, Z. F. Dai, J. Wang, Q. Cao, and Y. L. Zhang. Chiral induction, memory, and amplification in porphyrin homoaggregates based on electrostatic interactions. Chemphyschem, 10(6):954-962, 2009.

[17] K. Adachi, S. Tanaka, S. Yamazaki, H. Takechi, S. Tsukahara, and H. Watarai. Chirality induction and amplification in methylene blue h-aggregates via d- and l-phenylalanine pre-adsorbed on the tungsten oxide nanocolloid surface. New Journal of Chemistry, 36(11):2167-2170, 2012. 
[18] M. R. Watry and G. L. Richmond. Orientation and conformation of amino acids in monolayers adsorbed at an oil/water interface as determined by vibrational sum-frequency spectroscopy. Journal of Physical Chemistry B, 106:12517-12523, 2002.

[19] H. Takechi and H. Watarai. Chiral analysis of amino acids by synergistic heteroaggregation with porphyrins at liquid-liquid interface. Chemistry Letters, 40(3):303-305, 2011.

[20] E. C. Griffith, E. M. Adams, H. C. Allen, and V. Vaida. Hydrophobic collapse of a stearic acid film by adsorbed l-phenylalanine at the air-water interface. Journal of Physical Chemistry B, 116(27):78497857, 2012.

[21] C. Alonso, R. W. Gurney, R. Eliash, S. C. Hong, Y. R. Shen, T. R. Jensen, K. Kjaer, O. Konovalov, M. Lahav, and L. Leiserowitz. A crystalline Langmuir monolayer designed as a template for selective intercalation of water soluble r-amino acids. Crystal Growth $\&$ Design, 3(5):683-690, 2003.

[22] T. Michinobu, S. Shinoda, T. Nakanishi, J. P. Hill, K. Fujii, T. N. Player, H. Tsukube, and K. Ariga. Langmuir monolayers of a cholesterol-armed cyclen complex that can control enantioselectivity of amino acid recognition by surface pressure. Physical Chemistry Chemical Physics, 13(11):4895-4900, 2011.

[23] M. R. Palmer, J. M. Hagerman, L. M. Matano, K. M. DeWitt, and Y. J. Zhang. Thermodynamic analysis and fluorescence imaging of homochiral amino acid-amino acid interactions at the air/water interface. Journal of Colloid and Interface Science, 408:235-241, 2013.

[24] C. Moran, A. Pinazo, L. Pérez, P. Clapés, R. M. Angelet, and R. M. Infante. Arginine-based surfactants. Green Chemistry, 6:233-240, 2004.

[25] J. Kyte and R. F. Doolittle. A simple method for displaying the hydropathic character of a protein. Journal of Molecular Biology, 157(1):105-132, 1982.

[26] C. A. Schneider, W. S. Rasband, and Eliceiri K. W. Nih image to imagej: 25 years of image analysis. Nature Methods, 9:671-675, 2012.

[27] S. Haldar and S. K. Maji. Role of non-covalent interactions in the molecular organization of n-nhexadecanoyl amino acid amphiphiles with hydrophobic c-side chains in tris buffer (ph 9.3). Colloids and Surfaces A, 420:10-21, 2013.

[28] M. R. Infante, A. Pinazo, and J. Seguer. Non-conventional surfactants from amino acids and glycolipids: Structure, preparation and properties. Colloids and Surfaces A, 123-124:49-70, 1997.

[29] S. Trabelsi, S. Zhang, T. R. Lee, and D. K. Schwartz. Linactants: Surfactant analogues in two dimensions. Physical Review Letters, 100:037802, 2008.

[30] J. M. Ribó, J. Crusats, F. Sagués, J. Claret, and R. Rubires. Chiral sign induction by vortices during the formation of mesophases in stirred solutions. Science, 292(5524):2063-2066, 2001.

[31] O. Arteaga, A. Canillas, J. Crusats, Z. El-Hachemi, J. Llorens, E. Sacristan, and J. M. Ribó. Emergence of supramolecular chirality by flows. Chemphyschem, 11(16):3511-3516, 2010. 
Table 1: Abbreviations, chemical structures and hydropathy indexes of the studied amino acids ordered alphabetically. Last column indicates the measured $e_{C W}$ values with L-amino acids in the subphase at c.a. $1 \mathrm{mg} \mathrm{l}^{-1}$. The hydropathy index of an amino acid is a measure of the hydrophobic properties of its side chain [25], the more positive the more hydrophobic is the amino acid.

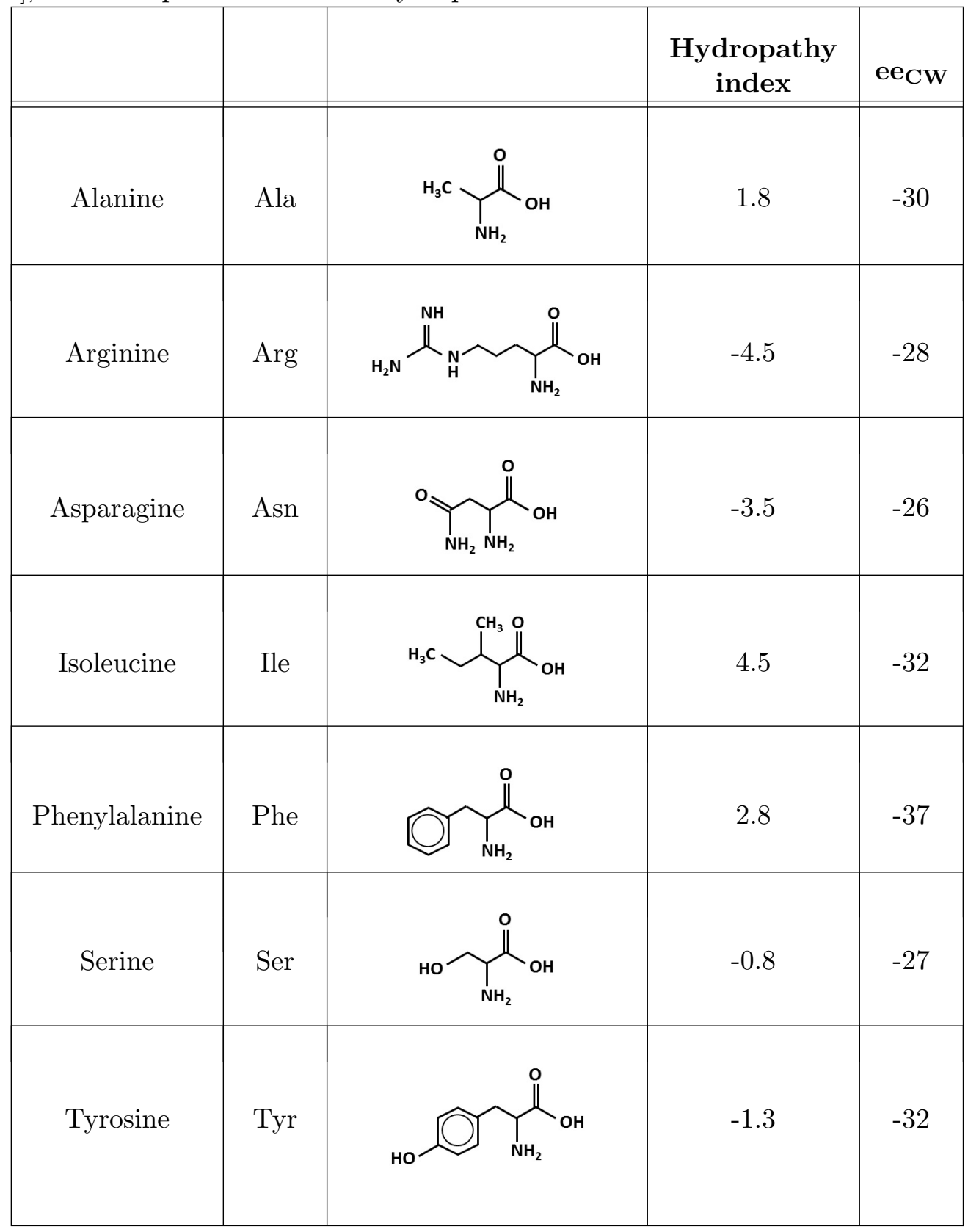

